\title{
Choosing cases and controls in neurological research
}

At first sight, case-control studies seem to be straightforward enough. All one has to do is compare some characteristic of cases of a particular disease with that of controls who do not have the disease. The characteristic to be compared may be a genetic marker, a physiological variable or a measure of past exposure to a known or suspected risk factor. But the apparent simplicity of the method disguises the real difficulties of choosing cases and appropriate controls and also in ensuring the unbiased measurement of the variable being investigated. Many case-control studies end up being hard to interpret because these problems were not fully appreciated when the work was being planned.

In case-control studies of neurological disease it is usually necessary to recruit prevalent (that is, existing) cases of disease rather than incident (that is, new) cases. Investigators who restricted themselves to new cases would find it hard to obtain enough people to achieve adequate statistical power for their studies. Unfortunately, the use of prevalent cases can lead to bias because patients with severe disease and reduced survival have less chance of being recruited than patients with milder disease and longer survival. If an association between disease and the variable being investigated is found, the correct explanation may not be that the variable influences the occurrence of the disease but that it affects how long patients survive. This source of bias may be part of the reason why studies of the association of certain HLA types with risk and severity of disease in patients with multiple sclerosis have produced conflicting results.

A more common problem with using prevalent cases in investigations of chronic disease arises because the presence of disease modifies some aspect of physiology or way of life of the cases. Differences between cases and controls may then be a result of having the disease rather than anything to do with the cause of the disease. The results of a case-control study of calcium metabolism in Alzheimer's disease, showed that cases tended to have lower plasma concentrations of vitamin D than controls. The likely explanation is not that vitamin $\mathrm{D}$ protects against Alzheimer's disease but only that demented patients spend less time outdoors exposed to sunlight.

Even if it proves possible to use newly diagnosed cases of disease, uncertainties about the correct interpretation of the results may remain. No case can be entered into a study unless a diagnosis has been made. The chances of a neurological condition being correctly diagnosed are unlikely to be uniform throughout the population. If the factors that determine the likelihood of diagnosis are related in some way to the variable being investigated, spurious associations might arise. In a case-control study designed to investigate whether urban dwellers are at greater risk of motor neuron disease than those living in a rural environment, a positive result might be found because people living in towns have easier access to neurological centres and, as a result, have a better chance of their condition being diagnosed.

Choice of an appropriate control group is a central part of the design of a case-control study. There are two principles to bear in mind: 1) for the variables under investigation, controls should be representative of the population from which the cases were obtained and 2) it must be possible to measure the variables in controls with the same degree of accuracy as for cases. Investigators who select controls haphazardly from colleagues, junior staff or medical students can rarely meet either of these requirements.

In hospital-based studies, patients with other diseases are frequently used as a convenient source of controls. Where the study relies on subjects remembering some past event this has the advantage that measuring the variable of interest can often be made compared with that for cases. A danger in studies that depend on recall to assess exposure to a putative risk factor is that, because of a natural interest in trying to understand why they have become ill, cases are more motivated to search their memories than controls. If the controls are also ill, they too will be seeking an explanation for their disease. The main weakness of using patients with other diseases as controls is that they may not be representative of the population from which the cases were drawn. For example, in a study of occupational exposures in patients with Parkinson's disease, it would be a mistake to select controls from those attending a neurological outpatient clinic. Such a control group would inevitably contain patients whose disease affected the sort of jobs in which they could be employed.

An alternative to using other patients as controls is to select controls from the general population. In the United Kingdom, where almost everyone is registered with a general practitioner, the records of Family Practitioner Committees can be used as the sampling frame. A standard system can also be used to select controls from the same general practice list or from the same neighbourhood as cases. Controls selected from the general population are likely to be more representative of the population from which the cases originated than if controls with other diseases are used. Even so, biases may occur if there is a poor response rate; the sort of people who agree to cooperate with research could be very different from those who refuse.

Often, the major problem when using controls selected from the general population, is ensuring that the variable under investigation is measured in the same way for them as it is in the cases. Subjects selected from the community will usually be less inclined to put time and effort into helping with a study than cases. They might, for example, be less meticulous in completing a questionnaire or in complying with instructions about avoiding food or alcohol before a blood sample is taken. This could lead to a systematic difference in the results from cases and controls that had nothing to do with the disease itself. A way around these difficulties is to use two control groups, one derived from the community and the second from patients with other diseases.

Studies are frequently reported in which the number of cases exceeds the number of controls. Although there is no need for the size of the two groups to be identical, the power 
of a study is severely reduced if there is a substantial deficit in the number of controls. Indeed, where it is difficult to find enough cases-a situation that is common when investigating neurological disease-increasing the number of controls to two or three per case can be the most cost- effective way to improve statistical efficiency.

Many investigators feel it necessary to match cases and controls for age, sex and many other factors as possible. Matching may seem intuitively to be a good way of minimising the confounding effect of determinants of disease other than the variable under study but it is not always advantageous. Once a factor has been matched in a case-control study, it is no longer possible to estimate the magnitude of the effect of that factor on the risk of disease. Another drawback is that matching increases the amount of time and effort required to find a suitable control. Many potential controls may have to be screened to find one with the same characteristics as the case. Decisions about whether or not to match, and what factors to match for, are far from simple. Advice from a statistician experienced in the analysis of case-control studies can be extremely valuable. A statistician may be able to suggest analytical techniques that can separate out the effect of the variables of interest on the risk of disease without the need for matching. If matching is undertaken, it must be carried out assiduously; there is usually little point, for example, in matching individual cases and controls for age less closely than within five years. It is also important to realise that any benefits of matching will be lost if the analysis of the results fail to take account of the matched design. Even worse, analysing a matched study as if it were unmatched may well give the wrong answer if the factors matched for really are determinants of the disease.

There are many potential sources of bias in case-control studies and no simple set of rules exists for avoiding them. The most useful advice is to become aware of their existence. Sackett has compiled a catalogue of different types of bias and given numerical examples to show how seriously they may distort the results. ${ }^{1}$ Anyone planning a case-control study ought to read this list before the protocol reaches its final draft. More help with the design and analysis of case-control studies will be found in the other references given below. ${ }^{2-5}$

CN MARTYN

MRC Environmental Epidemiology Unit, Southampton General Hospital, Southampton

1 Sackett DL. Bias in analytic research. J Chronic Dis 1979;32:51-63. Schlesselman JJ. Case-control studies. Design, conduct, analysis. Oxford: Oxford University Press, 1982.

3 MacMahon B, Pugh TF. Epidemiology-principles and methods. Casecontrol studies. Boston: Little, Brown and Company, 1970.

4 Breslow NE, Day NE. The analysis of case-control studies. Statistical methods in cancer research, Vol 1 . Lyon: IARC Scientific Publications No methods

5 Rothman KJ. Modern epidemiology. Boston: Little, Brown and Company, 1986. 